\title{
REPRESENTATION AS A POISSON TRANSFORM
}

\author{
BY \\ HARRY POLLARD( $\left.{ }^{1}\right)$
}

It is the purpose of this paper to establish necessary and sufficient conditions that a function $f(x)$, defined for all real values of $x$, admit representation as a Poisson transform

$$
f(x)=\frac{1}{\pi} \int_{-\infty}^{\infty} \frac{d A(t)}{1+(x-t)^{2}}, \quad-\infty<x<\infty,
$$

where $A$ is a nondecreasing function. If we ask in addition that $A$ be bounded the problem has already been solved by Standish [2] in terms of an operator described in the paper [1]. The removal of the condition of boundedness seems to require techniques quite different from his. In $\$ 5$ we establish a result concerning harmonic function which may be of independent interest.

1. The function $\hat{f}(z)$. In [1] it is established that a function $f(x)$ defined by the formula (1) necessarily admits analytic continuation into the strip $|y|<1$ of the complex $z$-plane. If we denote the continuation of $f(x)$ by $f(z)$ then (1) continues to hold if $x$ is replaced by $z$.

It is also shown in [1] that if $f(x)$ is defined by (1) then the function $\hat{f}(z)$ defined by

$$
\hat{f}(z)=-\frac{1}{\pi} \int_{0+}^{\infty} u^{-2}[f(z+u)-2 f(z)+f(z-u)] d u
$$

exists for all real $z$ and also admits continuation into the strip $|y|<1$. It is important for the sequel to know that the formula (1.1) persists for complex values of $z$ in the strip $|y|<1$.

For this purpose it is clearly enough that the integral

$$
\int_{-\infty}^{\infty} \frac{f(z-u)}{1+u^{2}} d u
$$

converges uniformly in compact subsets of the strip $|y|<1$. Since (1) holds with $x$ complex it suffices to verify this uniform convergence for the integral

$$
I=\int_{-\infty}^{\infty} \frac{d u}{1+u^{2}} \int_{-\infty}^{\infty} \frac{d A(t)}{\left|1+(z-u-t)^{2}\right|} .
$$

We are assuming of course that $A$ is nondecreasing.

Received by the editors June 15, 1956.

(1) The research of this author was supported by the United States Air Force under Contract No. AF 18(600)-685 monitored by the Office of Scientific Research. 
After an inversion of the order of integration and a change of variable $I$ takes the form

$$
I=\int_{-\infty}^{\infty} d A(t) \int_{-\infty}^{\infty} \frac{d u}{\left[1+(t-u)^{2}\right]\left|1+(z-u)^{2}\right|} .
$$

If $z=x+i y$ lies in some compact subset $S$ of the strip $|y|<1$, then

$$
|y|^{2} \leqq 1-\epsilon^{2}, \quad|x| \leqq B,
$$

where $\epsilon$ and $B$ depend only on $S$. For such $z$

$$
\left|1+(z-u)^{2}\right| \geqq \epsilon^{2}+(x-u)^{2},
$$

so that $I$ is dominated by

$$
\int_{-\infty}^{\infty} d A(t) \int_{-\infty}^{\infty} \frac{d u}{\left[1+(t-u)^{2}\right]\left[\epsilon^{2}+(x-u)^{2}\right]} .
$$

Apart from a multiplicative factor this is the same as

$$
\int_{-\infty}^{\infty} \frac{d A(t)}{(t-x)^{2}+(1+\epsilon)^{2}}
$$

which converges uniformly in $|x| \leqq B$, since

$$
f(0)=\frac{1}{\pi} \int_{-\infty}^{\infty} \frac{d A(t)}{t^{2}+1}
$$

exists by hypothesis.

We have proved that the integral in (1.1) converges uniformly in compact subsets of the strip $|y|<1$.

2. The representation theorem. Let $f(z)$ be analytic in the strip $|y|<1$, and let $\hat{f}(z)$ be defined by (1.1). We define an operator $T_{t} f$ for $-1<t<1$ and $-\infty<x<\infty$ by

$$
\left(T_{i} f\right)(x)=\frac{1}{2}[f(x+i t)+f(x-i t)]+\frac{1}{2 i} \int_{x-i t}^{x+i t} \hat{f}(u) d u
$$

whenever it has meaning.

The main result can now be stated.

THEOREM 2.1. Let $f(x)$ be defined for all real values of $x$. In order that it admit the representation (1) with $A$ a nondecreasing function it is necessary and suffcient that

(i) $f(x)$ admits analytic continuation into the strip $|y|<1$ of the z-plane;

(ii) the integral in (1.1) converges uniformly in compact subsets of $|y|<1$ to a function $\hat{f}(z)$;

(iii) $\left(T_{t} f\right)(x) \geqq 0,0 \leqq t<1,-\infty<x<\infty$; 
(iv) $f(x+i y)=o\left(x^{2}\right),|x| \rightarrow \infty$, uniformly in the substrips $|y| \leqq \delta$ for each $\delta<1$.

3. Proof of the necessity. Suppose that $f(x)$ has the form (1). The necessity of condition (i) is established in [1] and that of (ii) in the preceding section of this paper.

We turn our attention to (iii). In view of the analyticity of $f$ and $\hat{f}$ in the strip $|y|<1,\left(T_{t} f\right)(x)$ can be written as

$$
\sum_{k=0}^{\infty}(-1)^{k} \frac{t^{2 k}}{(2 k) !} f^{(2 k)}(x)+\sum_{k=0}^{\infty}(-1)^{k} \frac{t^{2 k+1}}{(2 k+1) !} \hat{f}^{(2 k)}(x) .
$$

By formula (4.1) of [1] this is in turn equal to

$$
\frac{1}{\pi} \int_{-\infty}^{\infty} \frac{v d A(u)}{v^{2}+(x-u)^{2}},
$$$$
v=1-t
$$

for $0 \leqq t<1$. (iii) follows since $A$ is nondecreasing.

As for condition (iv), we shall establish it under the condition $x \rightarrow-\infty$; the proof for $x \rightarrow+\infty$ is similar. We begin by recalling formula (1.3) of [1] which states (if we set $z_{0}=0$ ) that $f(z)$ can be written in the form

$$
f(z)=f(0)-2 z \int_{-\infty}^{\infty} F(t) \frac{1-(t-z) t}{\left[1+(z-t)^{2}\right]^{2}} d t,
$$

where $F(t)$ is the bounded function

$$
F(t)=\frac{1}{\pi} \int_{-\infty}^{t} \frac{d A(u)}{1+u^{2}} .
$$

Since $|y| \leqq \delta<1$ it suffices by (3.1) to prove that

$$
\begin{aligned}
J(z) & \equiv \int_{-\infty}^{\infty} F(t) \frac{1-(t-z) t}{\left[1+(z-t)^{2}\right]^{2}} d t \\
& =o(x),
\end{aligned}
$$$$
x \rightarrow-\infty,
$$

uniformly in $|y| \leqq \delta . J(z)$ can be written as $K(z)-z L(z)$, where

and

$$
K(z)=\int_{-\infty}^{\infty} F(t) \frac{1-(t-z)^{2}}{\left[1+(z-t)^{2}\right]^{2}} d t
$$

$$
L(z)=\int_{-\infty}^{\infty} F(t) \frac{t-z}{\left[1+(z-t)^{2}\right]^{2}} d t .
$$

We shall prove that each of these functions is $o(1)$ as $x \rightarrow-\infty$, uniformly in $|y| \leqq \delta$, and thus will complete the proof of (iv).

In each of the integrals defining $K$ and $L$ let $z=x+i y$ and then make the 
change of variable $u=t-x$. We get

$$
K(z)=\int_{-\infty}^{\infty} F(u+x) \frac{1-(u-i y)^{2}}{\left[1+(u-i y)^{2}\right]^{2}} d u
$$

and

$$
L(z)=\int_{-\infty}^{\infty} F(u+x) \frac{u-i y}{\left[1+(u-i y)^{2}\right]^{2}} d u .
$$

Then, since $|y| \leqq \delta<1$,

$$
|K(z)| \leqq \int_{-\infty}^{\infty}|F(u+x)| \frac{1+(|u|+\delta)^{2}}{\left(u^{2}+1-\delta^{2}\right)^{2}} d u
$$

and

$$
|L(z)| \leqq \int_{-\infty}^{\infty}|F(u+x)| \frac{|u|+\delta}{\left(u^{2}+1-\delta^{2}\right)^{2}} d u .
$$

Because $F$ is bounded and $F(-\infty)=0$ each of these is $o(1)$ as $x \rightarrow-\infty$.

4. The sufficiency. Suppose now that conditions (i)-(iv) of the theorem hold.

It is a consequence of them that the integral

$$
\int_{-\infty}^{\infty} \frac{|f(u)|}{1+u^{2}} d u
$$

is finite. For by (ii) if we let $z=0$ the integral

$$
\int_{1}^{\infty} \frac{f(u)-2 f(0)+f(-u)}{u^{2}} d u
$$

converges and by (iii) with $t=0$ we have $f(u) \geqq 0,-\infty<u<\infty$. This establishes that (4.1) is finite.

Consequently the function $u(x, t)$ defined by

$$
u(x, t)=\frac{1}{\pi} \int_{-\infty}^{\infty} \frac{(-t) f(u)}{(x-u)^{2}+t^{2}} d u
$$

exists and defines a function harmonic for $t<0,-\infty<x<\infty$. Since $f$ is continuous $u$ takes on the boundary value $f(x)$ as $t \rightarrow 0-$, and since $f \geqq 0$ the function $u$ is non-negative for $t<0$.

By (i) and (ii) both $f$ and $\widehat{f}$ are analytic in the strip $|y|<1$. Consequently the function $\left(T_{t} f\right)(x)$ defined by $(2.1)$ is harmonic in the region $-1<t<1$, $-\infty<x<\infty$. We shall show in $\S 5$ that $u(x, t)$ is the harmonic continuation of $\left(T_{t} f\right)(x)$ into the half-plane $t<0$. Consequently the two functions together form a function $v(x, t)$ which is harmonic in $t<1$ and which by condition 
(iii) is non-negative for $t<1$. Hence by Herglotz' theorem for a half-plane $v(x, t)$ takes the form

$$
v(x, t)=K(1-t)+\frac{1}{\pi} \int_{-\infty}^{\infty} \frac{(1-t) d A(u)}{(1-t)^{2}+(x-u)^{2}}
$$

for $t<1$, where $A$ is nondecreasing and $K$ is a non-negative constant. If we let $t=0$ and use the fact that $v(x, 0)=u(x, 0-)=f(x)$ we obtain from (4.3) that

$$
f(x)=K+\frac{1}{\pi} \int_{-\infty}^{\infty} \frac{d A(u)}{1+(x-u)^{2}} .
$$

Also by (4.3)

$$
K=\lim _{t \rightarrow-\infty} \frac{v(o, t)}{1-t}=\lim _{t \rightarrow-\infty} \frac{u(o, t)}{1-t}
$$

so by $(4.2)$

$$
K=\lim _{t \rightarrow \infty} \int_{-\infty}^{\infty} \frac{f(u)}{u^{2}+t^{2}} d u .
$$

Since (4.1) is finite we must have $K=0$. (4.4) reduces to (1) and the proof of sufficiency is complete.

It remains only to establish the preceding italicized remark concerning harmonic continuation.

5. Proof of the harmonic continuation. This requires that for $-1<t<0$ we have $u(x, t)=\left(T_{t} f\right)(x)$, or replacing $t$ by $-t$ that for $0<t<1$ the following formula holds:

(5.0) $\frac{1}{\pi} \int_{-\infty}^{\infty} \frac{t f(u)}{(x-u)^{2}+t^{2}} d u=\frac{1}{2}[f(x+i t)+f(x-i t)]-\frac{1}{2 i} \int_{x-i t}^{x+i t} \bar{f}(u) d u$.

The verification of this constitutes the remainder of the paper. For future applications we state the result explicitly.

THEOREM 5.1. Under hypotheses (i), (ii) and (iv) of Theorem 2.1 and the finiteness of (4.1) the formula (5.0) holds in the region $0<t<1,-\infty<x<\infty$.

Let $x$ be fixed and define $f(x+u)=g(u),-\infty<u<\infty$. By (1.1) $\hat{f}(x+u)$ $=\hat{g}(u)$. Therefore it is enough to establish (5.0) when $x=0$. We write this out explicitly:

$$
\frac{1}{\pi} \int_{-\infty}^{\infty} \frac{t f(u)}{u^{2}+t^{2}} d u=\frac{1}{2}[f(i t)+f(-i t)]-\frac{1}{2 i} \int_{-i t}^{i t} \hat{f}(u) d u,
$$

for $0<t<1$.

To prove this write the left-hand side of (5.1) as the limit as $R \rightarrow \infty$ of 


$$
I(R)=\frac{1}{2 \pi i} \int_{-R}^{R}\left\{\frac{1}{u-i t}-\frac{1}{u+i t}\right\} f(u) d u
$$

which can be written as

$$
I(R)=\frac{1}{2 \pi i} \int_{-R-i t}^{R-i t} \frac{f(w+i t)}{w} d w+\frac{1}{2 \pi i} \int_{R+i t}^{-R+i t} \frac{f(w-i t)}{w} d w .
$$

We rewrite the first term on the right-hand side as follows. Consider the rectangular contour with vertices at $-R,-R-i t, R-i t, R$ and indented by a semi-circle of radius $\rho$ below the origin. Since $f(w+i t) / w$ is analytic in and on this contour we have

$$
\frac{1}{2 \pi i} \int_{-R-i t}^{R-i t}=\frac{1}{2 \pi i} \int_{-R-i t}^{-R}+\frac{1}{2 \pi i} \int_{R}^{R-i t}+\frac{1}{2 \pi i} \int_{C},
$$

where $C$ denotes the part of the contour running from $R$ to $-R$ and indented below the origin. Now let $\rho \rightarrow 0$. Then $\int_{C}$ becomes the principal value integral P.V. $\int_{-R}^{R}$ plus $2 \pi i$ times half the residue of $f(w+i t) / w$ at $w=0$. Hence

$$
\frac{1}{2 \pi i} \int_{-R-i t}^{R-i t} \frac{f(w+i t)}{w} d w=\frac{1}{2 \pi i} \int_{-R-i t}^{-R}+\frac{1}{2 \pi i} \int_{R}^{R-i t}+\frac{1}{2 \pi i} \text { P.V. } \int_{-R}^{R}+\frac{f(i t)}{2} .
$$

There is a similar formula for the second term on the right-hand side of (5.2). Adding the two we get

$$
\begin{aligned}
I(R)= & \frac{1}{2}[f(i t)+f(-i t)]+\frac{1}{2 \pi i} \text { P.V. } \int_{-R}^{R} \frac{f(w+i t)-f(w-i t)}{w} d w \\
& +\frac{1}{2 \pi i} \int_{-R-i t}^{-R} \frac{f(w+i t)}{w} d w+\frac{1}{2 \pi i} \int_{-R}^{-R+i t} \frac{f(w-i t)}{w} d w \\
& +\frac{1}{2 \pi i} \int_{R}^{R-i t} \frac{f(w+i t)}{w} d w+\frac{1}{2 \pi i} \int_{R+i t}^{R} \frac{f(w-i t)}{w} d w .
\end{aligned}
$$

According to hypothesis (iv) we can in the denominators of the last two integrals replace $w$ by $R$ and in the preceding two by $-R$, with an error of $o(1)$ as $R \rightarrow \infty$. Hence

where

$$
I(R)=\frac{1}{2}[f(i t)+f(-i t)]+I_{1}+I_{2}+I_{3}+o(1), \quad R \rightarrow \infty,
$$

$$
\begin{aligned}
& I_{1}=\frac{1}{2 \pi i} \mathrm{P} \cdot \mathrm{V} \cdot \int_{-R}^{R} \frac{f(w+i t)-f(w-i t)}{w} d w, \\
& I_{2}=-\frac{1}{2 \pi i R}\left\{\int_{-R-i t}^{-R} f(w+i t) d w+\int_{-R}^{-R+i t} f(w-i t) d w\right\}
\end{aligned}
$$


and

$$
I_{3}=\frac{1}{2 \pi i R}\left\{\int_{R}^{R-i t} f(w+i t) d w+\int_{R+i t}^{R} f(w-i t) d w\right\},
$$

respectively. Since $I(\infty)$ is the left-hand side of (5.1) what remains to be proved is that $L=I_{1}+I_{2}+I_{3}+o(1), R \rightarrow \infty$, where

$$
L=-\frac{1}{2 i} \int_{-i i}^{i t} \widehat{f}(u) d u .
$$

By formula (1.1) and an integration by parts we have

$$
\begin{aligned}
\hat{f}(z)= & \frac{1}{\pi R}[f(z+R)+f(z-R)]-\frac{1}{\pi} \int_{0+}^{R} \frac{1}{u}\left[f^{\prime}(z+u)-f^{\prime}(z-u)\right] d u \\
& +o(1), \quad R \rightarrow \infty,
\end{aligned}
$$

uniformly in compact subsets of $|y|<1$. Hence, integrating from $-i t$ to it we have

$$
\begin{aligned}
L= & -\frac{1}{2 \pi i} \frac{1}{R} \int_{-i t}^{i t} f(z+R) d z-\frac{1}{2 \pi i} \frac{1}{R} \int_{-i t}^{i t} f(z-R) d z \\
& +\frac{1}{2 \pi i} \int_{0+}^{R} \frac{1}{u}[f(i t+u)-f(i t-u)-f(-i t+u)+f(-i t-u)] d u \\
& +o(1), \quad R \rightarrow \infty .
\end{aligned}
$$

The third term on the right-hand side is the same as $I_{1}$. We shall identify the first with $I_{3}$, and the second with $I_{2}$. This will complete the proof.

The first term can be written

$$
\begin{aligned}
-\frac{1}{2 \pi i} \frac{1}{R}\left\{\int_{0}^{i t} f(z+R) d z+\int_{-i t}^{0} f(z+R) d z\right\} \\
=-\frac{1}{2 \pi i} \frac{1}{R}\left\{\int_{R-i t}^{R} f(w+i t) d w+\int_{R}^{R+i t} f(w-i t) d w\right\}
\end{aligned}
$$

which is clearly the same as $I_{3}$.

A similar argument shows the second term is the same as $I_{2}$.

\section{REFERENCES}

1. H. Pollard, The Poisson transform, Trans. Amer. Math. Soc. vol. 78 (1955) pp. 541-550.

2. C. Standish, On the representation of a function by a Poisson transform, Michigan Math. J. vol. 3 (1955-1956) pp. 95-104.

Cornell University, ITHACA, N. Y. 